\title{
Discussion
}

\section{Alzheimer Research Forum Live Discussion: Reducing the Risk of Alzheimer's Disease ${ }^{1}$}

\author{
http://www.alzforum.org/res/for/journal/transcript.asp?liveID $=43$
}

Participants: Bill Thies (Alzheimer's Association), Lucy Boyd (Boyd Intellectual Properties, Tennessee), Dave Morgan (University of South Florida), Deborah Blacker (Massachusetts General Hospital, Boston), Donna McPhie (McLean Hospital), Lewis Kuller (University of Pittsburgh), David Sinclair (Harvard Medical School), Gabrielle Strobel (Alzheimer Research Forum), June Kinoshita (Alzheimer Research Forum), John Trojanowski (University of Pennsylvania), Sally Frautschy (University of California, Los Angeles), Sam Sisodia (University of Chicago), Greg Cole (University of California, Los Angeles) Marcelle Morrison-Bogorad (National Institutes of Health, Bethesda, Maryland).

Gabrielle Strobel: Welcome, everyone, to today's discussion. Sam, could you perhaps summarize in a nutshell your findings relevant to today's chat? Probably not everyone is familiar with the latest twists in your work that make you a discussion leader for this topic.

Sam Sisodia: In a nutshell, we reported that male transgenic mice expressing familial Alzheimer disease (FAD)-linked amyloid- $\beta$ protein precursor $(\mathrm{A} \beta \mathrm{PP})$ and presenilin (PS) that were exposed to an "enriched environment" exhibited reductions in $\mathrm{A} \beta$ burden and levels of $\mathrm{A} \beta$ peptides - measures that were inversely correlated with level of physical activity (in this case, running).

Gabrielle Strobel: While we are waiting for John Trojanowski, I will start the conversation by inviting all discussion leaders to state briefly the major finding of their research area important to today's topic.

Dave Morgan: I suspect the results prompting my inclusion regarded the work with calorie restriction (CR). $\mathrm{CR}$ reduces amyloid deposition in $\mathrm{A} \beta \mathrm{PP}$ mice, a result observed in three separate laboratories [1,2]. My perspective is that much of the environmental manipula-

\footnotetext{
${ }^{1}$ Note: Transcript has been edited for clarity and accuracy.
}

tion is slowing or accelerating the aging process. This leads to greater or lesser production/clearance of $\mathrm{A} \beta$.

Sally Frautschy: We found that in the APPsw Tg2576 mice, lowering intake of the omega- 3 fatty acid, docosahexaenoic acid (DHA), and increasing the omega- 6 fatty acid, linoleic acid, increased the loss of excitatory postsynaptic markers and amyloid levels and reduced insulin degrading enzyme [3]. In contrast, supplementing with omega-3 DHA protected excitatory postsynaptic markers and reduced amyloid. All of these worked with late intervention, consistent with the epidemiology suggesting that increased intake of omega-3 can protect against the risk of Alzheimer's disease.

Gabrielle Strobel: Regarding Dave Morgan's data, David Sinclair, are you looking at amyloid deposition, as well?

David Sinclair: We have not looked at deposition yet, but we will.

Lewis Kuller: Our major interest has been studying the strong association among small vessel disease in the brain, white matter abnormalities, ventricular enlargement, focal brain atrophy, and risk of dementia: Are small vessel disease in the brain, secondary inflamma- 
tion, and "oxidative stress" major causes of dementia and AD pathology?

Deborah Blacker: Working primarily in genetics, I think of prevention mainly as using genes to characterize risk/identify a high-risk sample for intervention trials and also to identify protective alleles. However, I was probably asked as an epidemiologist who is beginning to compile a new Alzforum database on AD epidemiology that will summarize available data on risk and protective factors.

David Sinclair: We work on a set of genes called the sirtuins that seems to control the body's defenses against aging and disease. They are activated by caloric restriction, the diet that protects against neurodegenerative disease and aging. We also have small molecules that activate these pathways that can slow disease progression in an AD model - the p25 mouse.

Sam Sisodia: David, would you describe the phenotypes in the AD p25 mouse for me?

David Sinclair: The mice experience severe neurodegeneration 12 weeks after p25 is switched on - along with plaques, tangles, and behavioral/memory defects.

Sam Sisodia: David, plaques and tangles in the p25 mice?

David Sinclair: Sam, this data is early and not from my laboratory, but the p 25 model gets more interesting the more is known.

Gabrielle Strobel: Sam, they are Li-Huei Tsai's mice, in which p25 seems to have effects in synaptic biology. Brief induction improves learning; chronic induction leads to neurodegeneration.

Sally Frautschy: I am interested in the differences between the Jankowsky group's [4] and the Sisodia group's [5] data on effects of enriched environment on amyloid. Jankowsky's group got improved function with increased amyloid, but their paradigm had less exercise than Sisodia's group had. What does Sam think?

Sam Sisodia: Sally, 24 female mice in a cage is a formula for stress...

David Sinclair: For all, I suspect most people do not know yet that sirtuin activating compounds (STACs) are proving effective in a number of neurodegenerative disorders, and that this data should come out in 2006. We are testing our transgenic SIRT1 mice for their ability to slow AD, Huntington (HD), Parkinson disease (PD) and amyotrophic lateral sclerosis (ALS).

Lucy Boyd: David, regarding the sirtuins that are activated by caloric restriction, can you describe the level of restriction required for activation? In a normal-weight animal, does this require weight loss beyond a level of normality?

John Trojanowski: David Sinclair, this is puzzling as $\mathrm{AD}, \mathrm{PD}, \mathrm{HD}$, and so on, all have different underlying mechanisms of disease, and many of us are skeptical that anti-A $\beta$ therapies will ameliorate all neurodegenerative pathways in $\mathrm{AD}$, or tauopathies, so how do STACs work across so many diverse diseases with different etiologies? While all involve aging, there are some hereditary forms of AD and frontotemporal dementia with parkinsonism linked to chromosome 17 (FTDP-17) that begin in the third decade.

David Sinclair: John, it seems that SIRT1 is nondiscriminatory in terms of mechanism. I am not surprised that activating an ancient, conserved defense pathway has such broad effects. It fits with the idea that we are mimicking caloric restriction, which prevents (in rodents and dogs, at least) most diseases of aging. It may take a while to know how SIRT1 is doing it, but it seems very powerful.

John Trojanowski: David, but if you speak in detail to investigators on these diseases, some assert that it is the toxic gain of function, as in tangles, plaques, and Lewy bodies, that drives neurodegeneration, while in HD, the field seems to argue the inclusions are protective and other mechanisms underlie degeneration distinct from brain amyloidoses. So, how would an intervention that ameliorates toxic gains of function in tangles promote the protective effects of aggregates in HD?

David Sinclair: All, we do not yet know how many targets SIRT1 has, but it could be dozens - from p53 and heat shock proteins to DNA repair. All I can say for sure is that the animal model data I have seen from various laboratories is impressive.

Sally Frautschy: What about blueberries? Dave Morgan found some improved performance, but I did not in my model. Blueberry-fed animals infused with $\mathrm{A} \beta$ 
oligomers showed worse performance in spatial memory.

Dave Morgan: Sally, I am not as convinced regarding the blueberry data as with other effects we have seen.

Lucy Boyd: Sally, how long did you administer the blueberries?

Sally Frautschy: In collaboration with Jim Joseph and Barbara Hale, I fed animals blueberries using their protocol for 5 months, introducing amyloid oligomers the third month. Dave, I think that blueberries are better for normal aging of the brain, but not so good for amyloid-specific effects.

David Sinclair: Sally, regarding plant extracts, have you heard about xenohormesis - the idea that molecules produced by stressed plants activate beneficial pathways in animals to treat disease? You can find articles I have written [6].

June Kinoshita: David, very interesting. What do these plant stress molecules do in animals? What pathways do they activate, and in what disease models?

Sally Frautschy: In India, the wild versions of plants (turmeric) are thought to have better medicinal qualities than the bred ones. But curcumin is highest in plants with the fewest tubers... Maybe this does fit in with your hypothesis.

David Sinclair: June, the stress molecules in the plant turn on stress pathways (e.g., the sirtuins) and the plant is stress-resistant. We believe that we (and most life forms) have evolved to pick up on the chemical stress cues produced by stressed plants, causing us to mount a defense response in anticipation of loss of food supply or other adversity. We see these molecules activate numerous pathways associated with better health and disease resistance; most of this is unpublished, but it is in accordance with the xenohormesis hypothesis. It means one small molecule can hit numerous beneficial targets, and it is not just luck; we evolved that way.

June Kinoshita: David, that is fascinating. Will there be a publication on this in the near future?

Greg Cole: David, the heat shock system seems to be upregulated with xenohormesis and it likely slows the rate of misfolded protein aggregate accumulation.
Gabrielle Strobel: David, this sounds fascinating but fairly non-specific. What is the cost to the organism of activating this stress response, especially long-term (i.e., side effects of such compounds)?

David Sinclair: Gabrielle, about long-term effects: We have a study that is 2 years on now, having treated mice with a STAC, and there are no apparent ill effects, only benefits so far. We also know that SIRT1 is activated by caloric restriction, and that having this protein elevated for the life of the rodent is beneficial in a number of disease areas.

Sally Frautschy: I am interested in the possibility that omega-3 fatty acids may not be protective for those with ApoE4, as suggested by epidemiology. I am interested in the difference between DHA and eicosapentanoic acid (EPA) omega-3 fatty acids. We have only tested DHA from algae. I think there needs to be a clinical trial to test DHA plus EPA versus DHA alone. EPA competes for DHA incorporation in the membrane so it may prevent DHA replenishment. However, EPA has anti-platelet effects and is a better anti-inflammatory in the periphery, and may be better for heart disease prevention.

John Trojanowski: David Sinclair, can one get the same effects with red wine rich in resveratrol?

Gabrielle Strobel: David, one last question: Are resveratrol and other SIRT inducers in foods such as wine and pomegranate potent enough to have a neuroprotective effect merely through dietary changes?

Sally Frautschy: I, personally, will take the curry. We measure curcumin in brain and show that it is high enough to reduce oligomer aggregation. Has anyone measured pomegranate or resveratrol in the brain to see if dietary doses reduce aggregation of amyloid?

David Sinclair: John, I sincerely doubt wine has enough resveratrol. In the mouse studies done at Harvard and with our collaborators at NIH, we use pure compound and it is at higher concentrations than you can get from red wine. There is a company called Sirtris that I co-founded in 2004 which has found novel SIRT1 activators that could have far better effects than resveratrol.

Gabrielle Strobel: David Sinclair, are you specifically targeting AD with your preclinical assays of those compounds (other than the p25 mice)? 
David Sinclair: Gabrielle, we have about 15 different experiments, mostly in the neurodegenerative area but also cancer, stem cells, aging, cardiovascular, metabolic... Lots of dollars are needed.

John Trojanowski: In general terms, one place to start is with some of the take-away messages from the Jedrziewski review of literature on lifestyle practices to reduce risk of dementia. Many of these practices come from studies by the folks here. They include lifestyles or activities that increase cognitive activity: education, exercise, avoiding head injury, good nutrition with a diet rich in antioxidants and that promotes low cholesterol. Are these agreed upon? Are there others not listed here?

Sam Sisodia: Environmental enrichment (EE) might be considered an analogue of several prospective and retrospective studies suggesting an important role for exercise and mental activity in reducing the risk for $\mathrm{AD}$ and cerebrovascular disease. Our microarray data have been quite informative and reveal upregulation of genes involved in neuroprotection, $\mathrm{A} \beta$ sequestration (transthyretin [TTR]), and synaptic plasticity. Indeed, our recent, unpublished data indicate that our $\mathrm{A} \beta \mathrm{PP} / \mathrm{PS}$ mice placed in a TTR $+/-$ background show earlier onset of deposition and enhanced $\mathrm{A} \beta$ burden in older animals.

Gabrielle Strobel: Sam, I understand the transthyretin connection but did not get that entirely. What is the meaning? Too much iron in brain accelerates amyloid deposition?

Sam Sisodia: Gabrielle, it has nothing to do with iron. TTR is a known $\mathrm{A} \beta$ binding/sequestering protein. Its normal function in brain is not fully known, but it is expressed in the choroid at high levels. It is probably involved in moving highly lipophilic molecules across the blood-brain barrier (BBB), but it is still a mystery why it is so highly upregulated in mice that have EE. We also see very high expression in $\mathrm{Tg} 2576$ mice at 6 months [7] well before pathology.... Is this a protective mechanism to sequester toxic $\mathrm{A} \beta$ species?

Sally Frautschy: TTR is a thyroid hormone transport protein induced by fish oil. Dmitry Goldgaber shows it is important in clearing amyloid from brain [8].

John Trojanowski: To the participants here, I ask if there is consensus around some of the briefly stated measures above or if there are disagreements or other activities to consider. Also, what research is needed to validate some of these strategies to reduce risk of dementia so they can be recommended to the public?

Lewis Kuller: I doubt whether any of the variables listed by John are really related to the etiology of dementia but probably just delay diagnosis. They are similar to risk factors for good health, prevention of heart disease, cancer, diabetes, and arthritis.

June Kinoshita: Lewis raises a central point. Why do the same risk factors pop up for all of these diseases of aging?

Dave Morgan: June, it is conceivable that they are modifying the overall rate of aging, and this larger process is setting the stage for disease.

Deborah Blacker: John (on the list of lifestyle factors) and Lewis, I agree. The best support is for those that clearly reduce cardiovascular risk (physical exercise, and cholesterol and weight reduction), and, of course, avoiding head injury is a good idea. I suspect education is very non-specific, and that mental activity may be a marker for education, and general physical and mental health.

Marcelle Morrison-Bogorad: John and Deb, the NIH (NIA, NINDS, NIMH) have had a Cognitive and Emotional Health Project going for several years now, one of the leaders being Molly Wagster of the NIA.

John Trojanowski: Yes, Lewis, these are all data from association studies, and they do not imply cause and effect, and the benefits do extend beyond brain aging or dementia. However, in the face of an obesity epidemic when folks like Jau Olshansky argue there will be a reversal in longevity trends, there still is a great need to demonstrate how these lifestyle activities might work and which can become preventatives rather than merely association findings $[9,10]$.

Greg Cole: Lewis, since the risk factors for AD strongly overlap those for heart disease, and along with cancer, arthritis, and aging they all have chronic inflammatory components, it is quite possible that they do relate to etiology.

Deborah Blacker: Greg, I agree that the cardiovascular risk factors may have the strongest link to etiology, but the mechanism remains unclear. 
Greg Cole: Deborah, there are likely multiple mechanisms of interest. At this point, all of the genetic and epidemiological risk factors seem to impinge on amyloid metabolism, but the details remain vague. It would be nice to know enough about mechanism to understand which of the protective strategies can be made to synergize.

Gabrielle Strobel: Greg, thanks, I was wondering the same thing. It seems that many of those factors affect amyloid metabolism. Is that really so, or does it merely appear to be so because people tend to check around the leading hypothesis first? Do studies such as Sam Sisodia's microarrays and David Sinclair's work with sirtuin inducers point to entirely different pathways?

Lewis Kuller: If cholesterol and exercise are important risk factors, the Japanese should have low rates.

Greg Cole: Lewis, it is true that one would expect lower rates based on environmental risk factors, but they live longer and do not die of cardiovascular disease (CVD), so the situation is complicated by competing causes, other diseases, and risk factor interactions. For example, in Japan ApoE4 seems to confer less risk than in Western populations. Why?

Deborah Blacker: Lewis, but the Japanese have lots of high blood pressure. Do you recall overall level of cardiovascular risk? I thought it was relatively high.

June Kinoshita: Regarding Gabrielle's question, I was wondering the same thing. How many other things have people looked at in the various mouse experiments, for example, white matter pathology, inflammation, insulin signaling, etc.?

Deborah Blacker: Greg, I share your sense that the ApoE4 risk is complicated by competing risk and interactions, and do not know about the specifics of cardiovascular disease risk in Japan to support a strong hypothesis. One possibility would relate to minor strokes that might make an AD diagnosis less likely (even if AD pathology is indeed present).

Greg Cole: Deborah, the autopsy rate is very low in Japan, so it makes you wonder how many minor stroke cases have mixed etiology.

Deborah Blacker: Greg, I suspect it is high, since it is high here, and rates of pure vascular dementia are low.
Lewis Kuller: Greg, age-specific AD pathology or magnetic resonance imaging (MRI) and positron emission tomography (PET) findings should be different among older Japanese versus Americans, etc. Life expectancy for Japanese age 65-70 is not very different from that in the US. The key is to find populations which have both low rates of clinical AD and dementia and low prevalence of pathology and/or MRI and PET abnormalities and determine the unique characteristics of such populations, as was done for coronary heart disease (CHD), stroke, diabetes, and many cancers.

Lucy Boyd: Lewis, I agree completely. Regarding education and cognitive activity as preventative measures, if this is so, does anyone have an opinion on whether it is due to a distinct difference made in the brain by learning, or if there is more reserve to lose before symptoms are noted?

Marcelle Morrison-Bogorad: Greg, we are funding an autopsy program by Lon White on Japanese in Hawaii, following up on the National Heart, Lung, and Blood Institute (NHLBI) cardiovascular study there. There are lots of interesting data on mixed dementias and those of unknown etiology.

June Kinoshita: Lewis, are there any populations that might meet your criteria?

Deborah Blacker: Lewis and Lucy, such a population would be great to study, but does one exist?

Greg Cole: Lewis, what group has the lowest dementia rates? What about Seventh-day Adventists? Maybe the Japanese need red wine instead of sake.

Lucy Boyd: I have noted the Seventh-day Adventists do have high rates of various types of dementia, probably due to a deficit of amino acids, in my opinion.

Sally Frautschy: Seventh-day Adventists do not eat enough protein and no omega-3 fatty acid (no DHA).

June Kinoshita: Regarding low-dementia populations, it would probably have to be one with a markedly different diet. Eskimos? Masai?

Deborah Blacker: June, again, I am skeptical that there is such a population, and there are a lot of methodological issues, both regarding assessment and regarding survival/competing risks for death and for disease, 
that would have to be addressed for you to be confident you had found one.

Bill Thies: Marcelle and Lewis, since there is little in the way of preventative trials in $\mathrm{AD}$, is it possible we can do trials in $\mathrm{AD}$ in the traditional way like those prevention trials for cardiovascular $(\mathrm{CV})$ disease. If we cannot, who has the next great methodology?

Deborah Blacker: Bill, the first big prevention trial, with a huge price tag, was halted because of risk. The vitamin $\mathrm{E}$ prevention trials with $\mathrm{AD}$ as secondary outcome were also halted. What would be next, and who will fund it? Anyone in this group?

Greg Cole: Bill, is it possible to try to get a prevention trial with an intervention likely to work for more than one disease of aging? For example, colon cancer, atherosclerosis, and AD all have inflammation as a significant risk factor. We are hoping that some type of polyphenolic antioxidant/nonsteroidal anti-inflammatory drug (NSAID) like curcumin or resveratrol could be used for more than one disease and have multiple agency sponsorship.

June Kinoshita: Greg's idea of trials aimed at slowing down general aging processes that affect risk for all kinds of chronic diseases of aging is interesting. How feasible is it, given that funders like to see impact on their specific diseases?

Deborah Blacker: Greg, this kind of thinking was behind the big vitamin E trials, too. It is a great idea, but there is reason for caution.

Bill Thies: Deborah, the results of AD prevention trials have been clear, so how do we do them faster and cheaper?

Deborah Blacker: Bill, doing them for mild cognitive impairment (MCI) was the way to do them faster and cheaper (I know, not cheap, but cheaper than the Alzheimer's Disease Antiinflammatory Prevention Trial (ADAPT), but the tradeoff is that you are not really doing prevention because the disease pathology is already in place.

John Trojanowski: Bill, there are so many potentially exciting ideas for intervention/prevention here, so how do we do what the March of Dimes did in the 1950s? Mobilize the American people to dedicate similar re- sources as those that cured polio to have a similar impact on aging-related neurodegenerative diseases?

Bill Thies: John, I do believe that one part of this is to begin a conversation with the public that lets them know we cannot solve the problem without their participation in clinical studies, both trials and natural history studies. If we can convince the public that they are a key part of finding the answers that are important to them, we will be part of the way to mobilization.

Marcelle Morrison-Bogorad: Bill, the Alzheimer's Disease Cooperative Study (ADCS) is working on home measures instead of clinical visits (far cheaper). And the Alzheimer Disease Neuroimaging Initiative (ADNI) is working on faster trials via research on surrogate markers.

June Kinoshita: Regarding faster and cheaper trials, we need antecedent biomarkers.

Deborah Blacker: John, great idea given the numbers involved and the level of fear. Can we pose this question to Marcelle, too, aware as we are that federal resources are thin, so we would still want moral support/matching funds?

Marcelle Morrison-Bogorad: Deborah, I would like to think that if a clinical trial (or two or three) were compelling, we would have additional non-NIH sponsors. But, however sponsored, we will probably have to go through pilot trials first to optimize all the methodology.

Lewis Kuller: Marcelle, I think you need to focus on geographic studies with imaging, and trials to prevent pathology using imaging and add-ons to trials, cardiovascular disease, etc.

Lucy Boyd: I believe the statins hold promise as ancillary prevention.

John Trojanowski: We also need a campaign like the March of Dimes that mobilized funds, but also volunteers in communities across the U.S. to eradicate a disease that, while terrible, does not begin to have the horrific consequences $\mathrm{AD}$ will have in the coming decades.

Sally Frautschy: John, yes, we need more campaigning. Trials with the strongest rationale (ibuprofen) cannot be done because of lack of money. We had to 
do naproxen because that company paid for placebo. Thus, we need donors to help complete costs of trials with the strongest rationale.

Greg Cole: Marcelle, is there any kind of cognitive testing that could be done with Internet testing at libraries under the supervision of a librarian? They are all connected.

Marcelle Morrison-Bogorad: Greg, I do not know. I will ask.

Deborah Blacker: Marcelle, pilot trials sound like a great option to help keep costs down. I hope the options for trials are compelling. We may have to kiss a lot of frogs, and pay for the privilege before we find our prince.

Sam Sisodia: Adding to John's notion, it is remarkable that over 25 percent of the US population is considered obese, and in Illinois, it is a staggering 28 percent... and with the increasing tightening of local budgets there is less exercise/gym periods in public schools (now only 45 minutes a week in Chicago public schools), so I would suggest that the first thing we instruct the public to do is to get off their sofas and eat fewer greasy burgers, etc. Prevention is really very simple and we need to educate the public, and under the auspices of the federal, state, and local governments.

Gabrielle Strobel: We have come to the end of the hour. Thanks to everyone.

\section{References}

[1] N.V. Patel, M.N. Gordon, K.E. Connor, R.A. Good, R.W. Engelman, J. Mason, D.G. Morgan, T.E. Morgan and C.E. Finch,
Caloric restriction attenuates Abeta-deposition in Alzheimer transgenic models, Neurobiol Aging 26 (2005), 995-1000.

[2] J. Wang, L. Ho, W. Qin, A.B. Rocher, I. Seror, N. Humala, K. Maniar, G. Dolios, R. Wang, P.R. Hof and G.M. Pasinetti, Caloric restriction attenuates beta-amyloid neuropathology in a mouse model of Alzheimer's disease, FASEB J 19 (2005), 659-661.

[3] F. Calon, G.P. Lim, T. Morihara, F. Yang, O. Ubeda, N. Salem, S.A. Frautschy and G.M. Cole, Dietary n-3 polyunsaturated fatty acid depletion activates caspases and decreases NMDA receptors in the brain of a transgenic mouse model of Alzheimer's disease, Eur J Neurosci 22 (2005), 617-626.

[4] J.L. Jankowsky, T. Melnikova, D.J. Fadale, G.M. Xu, H.H. Slunt, V. Gonzales, L.H. Younkin, S.G. Younkin, D.R. Borchelt and A.V. Savonenko, Environmental enrichment mitigates cognitive deficits in a mouse model of Alzheimer's disease, J Neurosci 25 (2005), 5217-5224.

[5] O. Lazarov, J. Robinson, Y.P. Tang, I.S. Hairston, Z. KoradeMirnics, V.M. Lee, L.B. Hersh, R.M. Sapolsky, K. Mirnics and S.S. Sisodia, Environmental enrichment reduces Abeta levels and amyloid deposition in transgenic mice, Cell 120 (2005), $701-713$.

[6] D.W. Lamming, J.G. Wood and D.A. Sinclair, Small molecules that regulate lifespan: evidence for xenohormesis, Mol Microbiol 53 (2004), 1003-1009.

[7] T.D. Stein and J.A. Johnson, Lack of neurodegeneration in transgenic mice overexpressing mutant amyloid precursor protein is associated with increased levels of transthyretin and the activation of cell survival pathways, J Neurosci 22 (2002), 7380-7388.

[8] A.L. Schwarzman, M. Tsiper, H. Wente, A. Wang, M.P. Vitek, V. Vasiliev and D. Goldgaber, Amyloidogenic and antiamyloidogenic properties of recombinant transthyretin variants, Amyloid 11 (2004), 1-9.

[9] S.J. Olshansky, D.J. Passaro, R.C. Hershow, J. Layden, B.A. Carnes, J. Brody, L. Hayflick, R.N. Butler, D.B. Allison and D.S. Ludwig, A potential decline in life expectancy in the United States in the 21st century, N Engl J Med 352 (2005), 1138-1145.

[10] S.J. Olshansky, B.A. Carnes, R. Hershow, D. Passaro, J. Layden, J. Brody, L. Hayflick, R.N. Butler, D.B. Allison and D.S. Ludwig, Misdirection on the road to Shangri-La, Sci Aging Knowledge Environ 2005 (2005), 15. 